\title{
Design of a Humanoid Robot Eye: Models and Experiments
}

\author{
Giorgio Cannata and Mirko D'Andrea \\ Mechatronics and Automatic Control Laboratory \\ Dept. of Communications, Computer and System Sciences, \\ University of Genova \\ Via Opera Pia 13, 16145 Genova, Italy \\ Email: cannata@dist.unige.it
}

\author{
Marco Maggiali \\ Mechatronics and Automatic Control Laboratory \\ Italian Institute of Technology, \\ University of Genova \\ Via Opera Pia 13, 16145 Genova, Italy \\ Email: marco.maggiali@unige.it
}

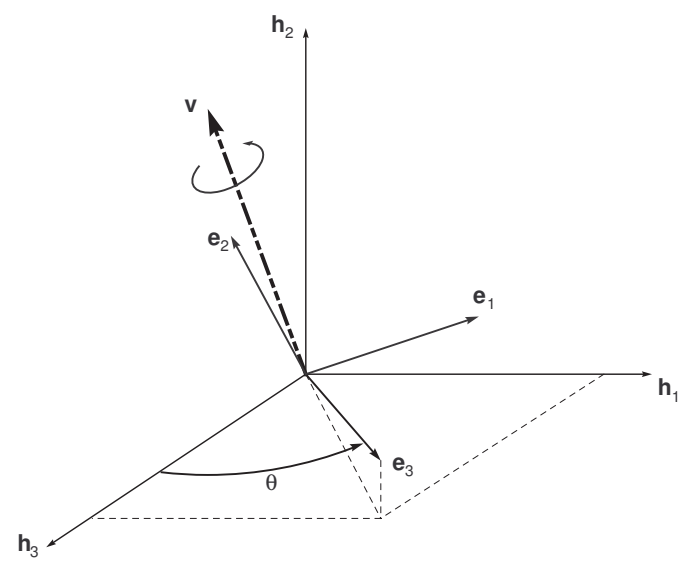

\begin{abstract}
Eye movements have the goal of optimizing visual perception, therefore the investigation of eye motion strategies play an important role in the design of humanoid robot eye systems. Saccades in humans and primates is a significant class of ocular motions, which obey the so called Listing's Law, which constrains the admissible eye's angular velocities and ensure zero torsion during motion. In this paper we present a model of the eye plant proving that Listing's Law implementation is strongly related with the geometry of the eye and its actuation system (extraocular muscles). The proposed model has been used to provide the guidelines for the design of a tendon driven humanoid robot eye. Experimental tests, presented in this paper, validate the model by performing a quantitative comparison of the performance of the robot eye with physiological data measured in humans and primates during saccades.
\end{abstract}

\section{INTRODUCTION}

Eye movements have the goal of optimizing visual perception, [1]. The way the eyes change their orientation may affect our perception of the world. In turn, it is widely accepted that visual feedback, as well as other sensory feedback (e.g. from vestibular system), play a major role in stimulating eye movements. Therefore, it is not surprising that to different vision strategies correspond significantly different types of eye motions.

Saccades represent a basic and important class of eye movements, [2], where the goal is that of reaching as fast as possible a target direction of fixation. During saccades the eye orientation is determined by a basic principle known as Listing's Law, which establishes the amount of eye torsion for each direction of fixation. Listing's Law has been formulated in the mid of the $19^{t h}$ century, but has been experimentally verified on humans and primates only during the last 20 years, [3]- [6]. Furthermore, Listing's Law has been recently found to be valid also during other types of eye movements such as smooth pursuit, [7].

A formal statement of Listing's Law can be formulated as follows.

Listing's Law: There exists a specific eye orientation with respect to the head, called primary position. During saccades any physiological eye orientation, with respect to the primary position, can be described by a unit quaternion $q$ whose (unit) rotation axis, $\mathbf{v}$, always belongs to a head fixed plane, $\mathcal{L}$
Fig. 1. Geometry of Listing compatible rotations: the finite rotation axis $\mathbf{v}$ must always belong to a head fixed plane $\mathcal{L}$. Without loss of generality $\mathbf{h}_{\mathbf{3}}$ define the direction of fixation at the primary position.

(Listing plane). The normal to plane $\mathcal{L}$ is the eye's direction of fixation at the primary position.

Figure 1 describes the geometry of a eye rotation compatible with Listing's Law. Vectors $\left(\mathbf{h}_{\mathbf{1}}, \mathbf{h}_{\mathbf{2}}, \mathbf{h}_{\mathbf{3}}\right)$ and $\left(\mathbf{e}_{\mathbf{1}}, \mathbf{e}_{\mathbf{2}}, \mathbf{e}_{\mathbf{3}}\right)$ represent head and eye fixed reference frames respectively. Any physiological eye orientation can be obtained by rotating the eye by a finite angle $\theta$, about an axis $\mathbf{v}$ always belonging to a head fixed plane $\mathcal{L}$. Without loss of generality, in the following $\mathbf{h}_{\mathbf{3}}$ will be assumed orthogonal to $\mathcal{L}$, and define the direction of fixation at the primary position.

During saccades both $\theta$ and $\mathbf{v}$ are time functions conveniently described by a unit quaternion, [8]. However, as discussed in [8] and [9], in order to ensure that $\mathbf{v} \in \mathcal{L}$ at any time, the eye's angular velocity $\boldsymbol{\omega}$, must belong to a plane $\mathcal{P}_{\omega}$, passing through $\mathbf{v}$, and whose normal forms an angle of $\frac{\theta}{2}$ with the direction of fixation at the primary position, see figure 2. This property, directly implied by Listing's Law, is usually called Half Angle Rule, [10].

During a generic saccade the plane $\mathcal{P}_{\omega}$ is rotating with respect to both the head and the eye due to its dependency from $\mathbf{v}$ and $\theta$. This fact poses important questions related to 


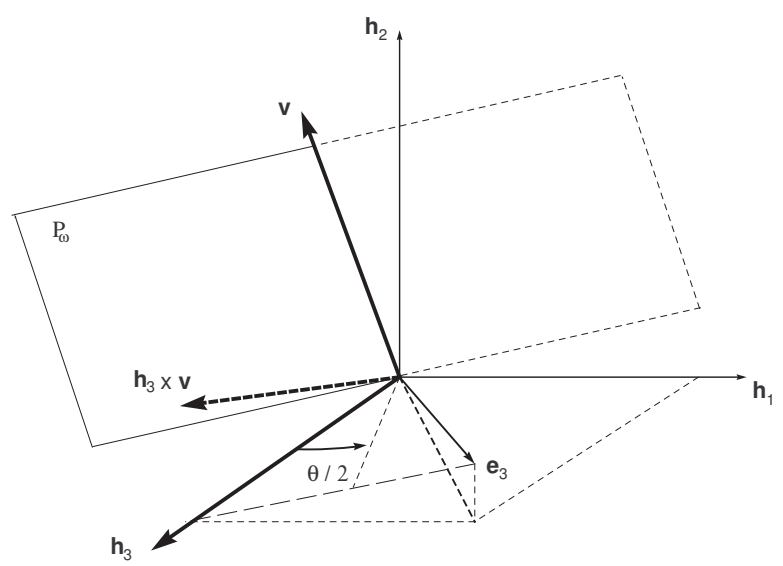

Fig. 2. Half angle rule geometry. The eye's angular velocity must belong to the plane $\mathcal{P}_{\omega}$ passing through axis $\mathbf{v}$.

the control mechanisms required to implement the Listing's Law, also in view of the fact that there is no evidence of sensors in the eye plant capable to detect how $\mathcal{P}_{\omega}$ is oriented.

Whether Listing's Law is implemented in humans and primates on a mechanical basis or requires an active feedback (neural) control action has been disputed among neurophysiologists in the past few years. The evidence of soft tissue within the orbit, called soft pulleys, [11] - [15], constraining the extra ocular muscles (EOMs), has suggested that the mechanics of the eye plant could have a significant role in the implementation of half angle rule and Listing's Law, [16][19], although counterexamples have been presented in the literature, [20], [21].

A model fully explaining the role of the geometry of the eye plant for implementing the Listing's Law has been proposed in [8] and [9]. In this paper the model is shortly discussed and guidelines to the design of a tendon driven robot eye are provided. These guidelines have been used to implement a prototype of humanoid robot eye, [22].

The major contribution of this paper is to show through simulative and experimental results that the proposed model is accurate enough to describe the eye movements observed in humans and primates. Furthermore, it is shown that the robot implementation has performances close to those predicted by the model. Finally, it is shown that the experimental tests performed on the robot prototype produce results comparable with the data measured on humans and primates during saccadic motions.

The structure of the paper is the following. In section II a model of the eye plant is presented, and some of its relevant geometric and kinematic properties are discussed in section III, including some guidelines used for the design of a tendon driven robot eye. In section IV, some important characteristics of the proposed models are exploited through simulation and experiments, and a comparison with data obtained from actual measured saccades is performed.

\section{EYE MODEL}

The eye in humans has an almost spherical shape and is actuated by six extra-ocular muscles (EOMs), [23]. Each EOM has an insertion point (IP) on the sclera, and is connected with the bottom of the orbit at the other end. Accordingly with the rationale proposed in [16] and [24], only the four rectii EOMs play a significant role during saccadic movements.

The motion of EOMs within the orbit is constrained by connective tissue, known as soft pulleys, whose physiologic role during ocular movements has been widely discussed, [11]- [15].

In [25] a complete 3D model of the eye plant including a non linear dynamics description of the EOMs has been proposed. This model has been extended in [26] and [27], including also a description of the soft pulleys as elastic suspensions (springs). However, this model require that the elastic suspensions perform particular movements in order to ensure that Listing's Law is fulfilled.

The model proposed in [8], and [9], and described in the following, is slightly simpler than the previous ones. In fact, it does not include EOMs dynamics since it can be shown that it has no role in implementing the Listing's Law; moreover, it models soft pulleys as fixed point-wise pulleys. It will be clear in the next section that this model despite its simplicity can be also used as a guideline for the design of humanoid tendon driven robot eyes.

The eye-ball is assumed to be modelled as a homogeneous sphere of radius $R$, having 3 rotational degrees of freedom (DOFs) about its center. EOMs are modelled as non-elastic thin wires, [16], connected to pulling force generators, [26].

Starting from the IPs placed on the eye-ball, the EOMs are routed through head fixed point-wise pulleys (PPs), emulating the soft-pulley tissue. The PPs are located on the rear of the eye-ball, and it will be shown that appropriate placement of the PPs and of the IPs has a fundamental role to implement the Listing's Law on a purely mechanical basis.

Let $O$ be the eye's center, then the position of the PPs can be described by vectors $\mathbf{p}_{\mathbf{i}}$, while, at the primary position IPs can be described by vectors $\mathbf{c}_{\mathbf{i}}$ assuming also that $\left|\mathbf{c}_{\mathbf{i}}\right|=R$.

When the eye is rotated about a generic axis $\mathbf{v}$ by an angle $\theta$ the position of the IPs can be expressed as:

$$
\mathbf{r}_{\mathbf{i}}=R(\mathbf{v}, \theta) \mathbf{c}_{\mathbf{i}} \quad \forall i=1 \ldots 4 .
$$

where $R(\mathbf{v}, \theta)$ is the rotation operator from the eye to the head coordinate systems.

Each EOM is assumed to follow the shortest path from each IP to the corresponding pulley, [13]; then, the path of the each EOM, for any eye orientation, belongs to the plane defined by vectors $\mathbf{r}_{\mathbf{i}}$ and $\mathbf{p}_{\mathbf{i}}$. Therefore, the torque applied to the eye by the pulling action $\tau_{i} \geq 0$ of each EOM is given by:

$$
\mathbf{m}_{\mathbf{i}}=\tau_{i} \frac{\mathbf{r}_{\mathbf{i}} \times \mathbf{p}_{\mathbf{i}}}{\left|\mathbf{r}_{\mathbf{i}} \times \mathbf{p}_{\mathbf{i}}\right|} \quad \forall i=1 \ldots 4
$$

From expression (2), it is clear that $\left|\mathbf{p}_{\mathbf{i}}\right|$ does not affect the direction or the magnitude of $\mathbf{m}_{\mathbf{i}}$, so we shall assume in the following that $\left|\mathbf{p}_{\mathbf{i}}\right|=\left|\mathbf{c}_{\mathbf{i}}\right|$. Instead, the orientation of the 


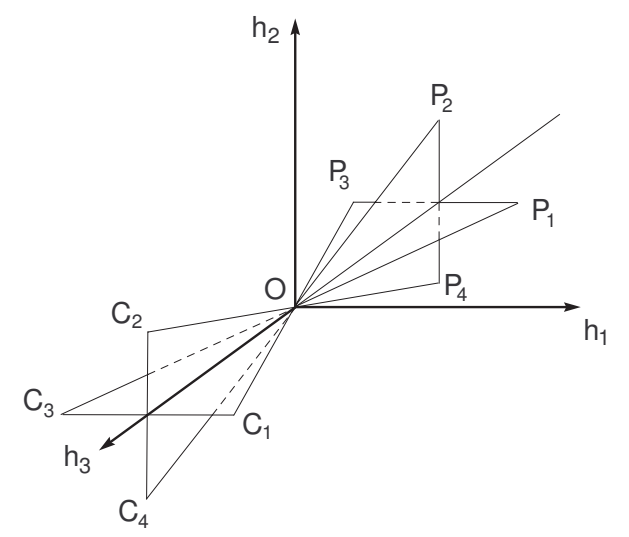

Fig. 3. Relative position of pulleys and insertion points when the eye is in the primary position.

vectors $\mathbf{p}_{\mathbf{i}}$, called principal directions (PDs), are extremely important.

In fact, it is assumed that $\mathbf{p}_{\mathbf{i}}$ and $\mathbf{c}_{\mathbf{i}}$ are symmetric with respect to the plane $\mathcal{L}$ which implies:

$$
\left(\mathbf{v} \cdot \mathbf{c}_{\mathbf{i}}\right)=\left(\mathbf{v} \cdot \mathbf{p}_{\mathbf{i}}\right) \quad \forall i=1 \ldots 4, \forall \mathbf{v} \in \mathcal{L} ;
$$

and finally it is assumed that IPs are symmetric with respect to the fixation axis, i.e.:

$$
\left(\mathbf{h}_{\mathbf{3}} \cdot \mathbf{c}_{\mathbf{i}}\right)=\left(\mathbf{h}_{\mathbf{3}} \cdot \mathbf{c}_{\mathbf{j}}\right) \quad \forall i, j=1 \ldots 4,
$$

and

$$
\left(\mathbf{c}_{3}-\mathbf{c}_{1}\right) \cdot\left(\mathbf{c}_{4}-\mathbf{c}_{2}\right)=0 .
$$

Figure 3 shows the relative position of IPs and PPs when the eye is in its primary position.

\section{Model Properties}

In this section we review the most relevant properties of the proposed model.

First, it is possible to show that for any eye orientation compatible with Listing's Law all the torques $\mathbf{m}_{\mathbf{i}}$ produced by the four rectii EOMs belong to a common plane passing through the finite rotation axis $\mathbf{v} \in \mathcal{L}$ (see [8] for proof).

Theorem 1: Let $\mathbf{v} \in \mathcal{L}$ be the finite rotation axis for a generic eye orientation, then there exists a plane $\mathcal{M}$ passing through $\mathbf{v}$ such that

$$
\mathbf{m}_{\mathbf{i}} \in \mathcal{M} \quad \forall i=1 \ldots 4
$$

A second important result is that the relative position of the IPs and PPs, at any Listing compatible eye's orientation, form a set of parallel vectors, as stated by the following theorem (see [8] for proof).

Theorem 2: Let $\mathbf{v} \in \mathcal{L}$ be the finite rotation axis for a generic eye orientation, then:

$$
\left(\mathbf{r}_{\mathbf{i}}-\mathbf{p}_{\mathbf{i}}\right) \times\left(\mathbf{r}_{\mathbf{j}}-\mathbf{p}_{\mathbf{j}}\right)=0 \quad \forall i, j=1 \ldots 4
$$

Finally, it is possible to show that planes $\mathcal{M}$ and $\mathcal{P}_{\omega}$ are coincident (see [8] for proof).

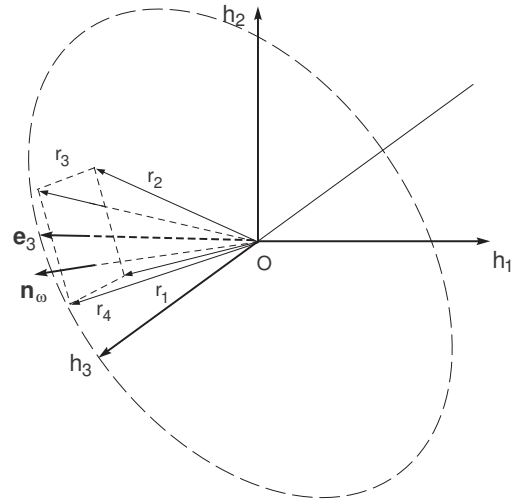

Fig. 4. When vector $\mathbf{n}_{\omega}$ belongs to the convex hull of vectors $\mathbf{r}_{\mathbf{i}}$ then rectii EOMs can generate any admissible torque on $\mathcal{P}_{\omega}$.

Theorem 3: Let $\mathbf{v} \in \mathcal{L}$ be the finite rotation axis for a generic eye orientation, then:

$$
\mathbf{m}_{\mathbf{i}} \in \mathcal{P}_{\omega} \quad \forall i=1 \ldots 4
$$

Remark 1: Theorem (3) has in practice the following significant interpretation. For any Listing compatible eye orientation any possible torque applied to the eye, and generated using only the four rectii EOMs, must lay on plane $\mathcal{P}_{\omega}$.

The problem now is to show, accordingly to formula (2), when arbitrary torques $\mathbf{m}_{\mathbf{i}} \in \mathcal{P}_{\omega}$ can be generated using only pulling forces. Theorem 2 states that $\mathbf{m}_{\mathbf{i}}$ are all orthogonal to a vector $\mathbf{n}_{\omega}$ normal to plane $\mathcal{P}_{\omega}$. Therefore, formula (2) can be rewritten as:

$$
\boldsymbol{\tau}=-\mathbf{n}_{\omega} \times\left(\sum_{i=1}^{4} \gamma_{i} \mathbf{r}_{\mathbf{i}}\right)
$$

where $\gamma_{i}=\frac{\tau_{i}}{\left|\mathbf{n}_{\omega} \times \mathbf{r}_{\mathbf{i}}\right|} \geq 0$ take into account the actual EOMs pulling forces. From formula (6), $\tau$ is orthogonal to a convex linear combination of vectors $\mathbf{r}_{\mathbf{i}}$. Then, it is possible to generate any torque vector laying on plane $\mathcal{P}_{\omega}$, as long as $\mathbf{n}_{\omega}$ belongs to the convex hull of vectors $\mathbf{r}_{\mathbf{i}}$, as shown in figure (4).

Remark 2: The discussion above shows that the placement of the IPs affects the range of admissible motions of the eye.

Accordingly with the previous discussion when the eye is in its primary position any torque belonging to plane $\mathcal{L}$ can be assigned. Assume now that, under the assumptions made in section II, a simplified dynamic model of the eye could be expressed as:

$$
I \dot{\omega}=\tau
$$

where $I$ is the inertia matrix of the eye, assumed to be proportional to the identity matrix. Assume the eye at time 0 to be in the primary position, with zero angular velocity (zero state). The EOMs can generate a resulting torque of the 


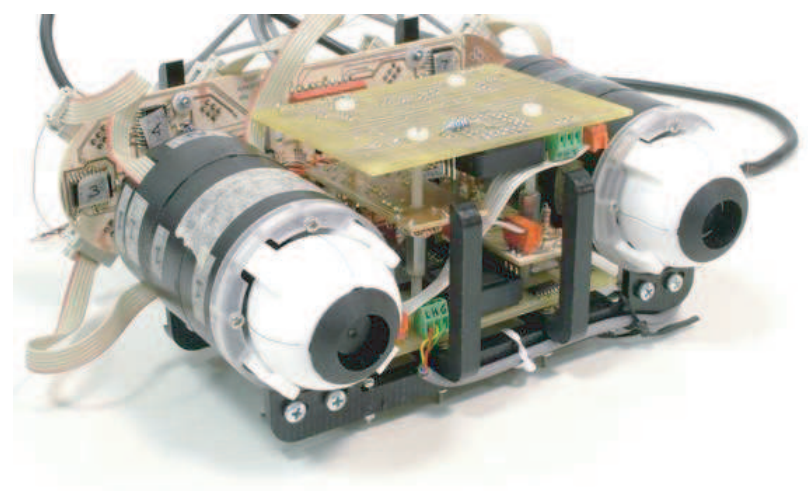

Fig. 5. Complete stereoscopic robot system

form:

$$
\boldsymbol{\tau}=\mathbf{v} \theta(t)
$$

where $\mathbf{v} \in \mathcal{L}$ is a constant vector and $\theta(t)$ a scalar control signal. Therefore, $\dot{\omega}$ and $\boldsymbol{\omega}$ are parallel to $\mathbf{v}$. Then, it is possible to reach any Listing compatible orientation, and also, during the rotation, the Half Angle Rule is satisfied.

Similar reasoning can be applied to control the eye orientation to the primary position starting from any Listing compatible orientation and zero angular velocity.

Remark 3: The geometric specifications provided in section II and the properties described in this section provided operational guidelines for the development of a tendon driven robot eye (MAC-EYE), [22]. Figure (5) shows the complete system including the embedded control electronics. Each eye is actuated by four independent DC motors driving tendons routed to the eye-ball. Embedded optical sensors provide feedback to control the mechanical tension of the tendons. The eye-ball is made of PTFE and is supported by a custom made bearing. Sliding pulleys emulating the geometry of the PPs discussed in the paper have been implemented, [22].

\section{Simulations And Experimental Tests}

Further analysis, beyond the theoretical results presented in the previous sections, has been carried out through simulative and experimental tests performed on a prototype of tendon driven robot eye, [22].

All the tests consisted of simulated (or experimental) consecutive saccadic motions (from a hundred for simulations up to a thousand, for the robot tests). At the end of each single saccade the quaternion $q=\left(\cos \frac{\theta}{2}, \mathbf{v} \sin \frac{\theta}{2}\right)$ describing the eye rotation has been measured. The vector part of the quaternion has been then projected on a head fixed reference frame $\left(\mathbf{h}_{1}, \mathbf{h}_{2}, \mathbf{h}_{3}\right)$ (being $\mathbf{h}_{3}$ orthogonal to Listing's plane), and scaled as follows:

$$
\begin{aligned}
& q_{H}=\frac{180}{\pi} \mathbf{h}_{\mathbf{1}} \cdot \mathbf{v} \sin \frac{\theta}{2} \\
& q_{V}=\frac{180}{\pi} \mathbf{h}_{\mathbf{2}} \cdot \mathbf{v} \sin \frac{\theta}{2} \\
& q_{T}=\frac{180}{\pi} \mathbf{h}_{\mathbf{3}} \cdot \mathbf{v} \sin \frac{\theta}{2}
\end{aligned}
$$

As suggested in [5], since within the oculomotor range $\sin \frac{\theta}{2} \approx$ $\frac{\theta}{2}$ then $q_{T}$ can be used to measure the amount of torsion of

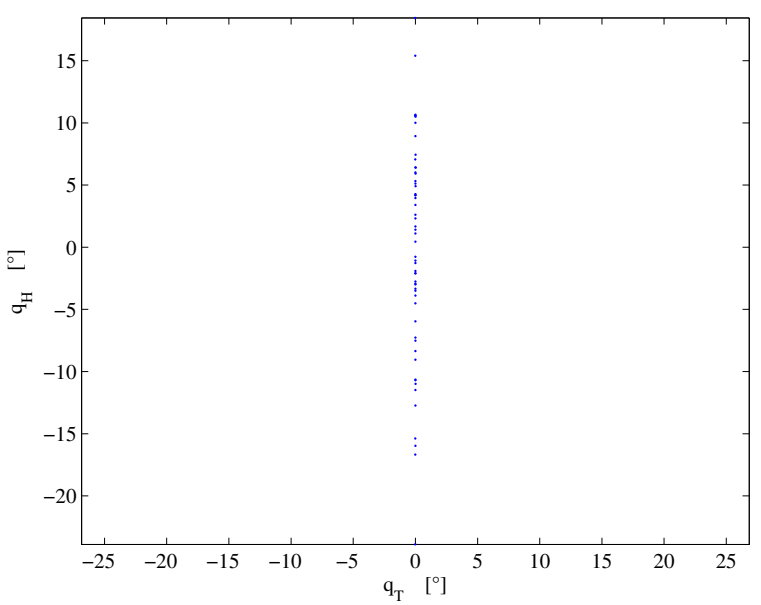

Fig. 6. Plane $\left(q_{T}, q_{H}\right)$. Each dot represents a single eye orientation. All the points correspond to zero torsion eye orientations as specified by Listing's Law.

the eye (in degrees); while, $q_{H}$ and $q_{V}$ represent the amount of vertical and horizontal rotation.

\section{A. Simulations}

The eye model has been implemented using Simulink accordingly with the geometric guidelines described in the previous sections and correspond to the model used for the design of the robot eye prototype, [22]. In particular the eye ball is a sphere with a mass of $50[\mathrm{~g}]$, and diameter of $38.1[\mathrm{~mm}]$. The IPs form an angle of $55[\mathrm{deg}]$, corresponding to the configuration of EOMs in the human eye. On the basis of the conditions stated using formula (6), this configuration ensures an oculomotor range larger than $45[\mathrm{deg}]$ in all directions. Principal directions are established accordingly. Viscoelastic actuation forces (linear spring-damper mechanism) have been used to model the action of the EOMs. The rotation of the eye is then obtained by changing the length the spring.

1) Nominal Model: the goal of this test has been to show that the model obeys Listing's Law for the geometry described in section II. Figure 6 shows the plane $\left(q_{T}, q_{H}\right)$ and it is clear that all the saccades produce eye rotations compatible with Listing' Law, i.e. $q_{T}=0$.

2) Perturbed Model: the goal of this test has been to measure the sensitivity of the model with respect to perturbations of the parameters. As a matter of fact fixed PPs are an idealization of the actual soft pulley tissue. In this case a hundred saccadic movements uniformly distributed within the range of rotation of the eye have been simulated. The position of the four PPs has been randomly perturbed from the principal directions by $2.5 \%$ with respect to the eye ball diameter.

The effect of the perturbations is a thickening of the Listing plane, but all the eye orientation are still distributed along a plane. This fact is confirmed also by the physiological evidence as discussed below. 


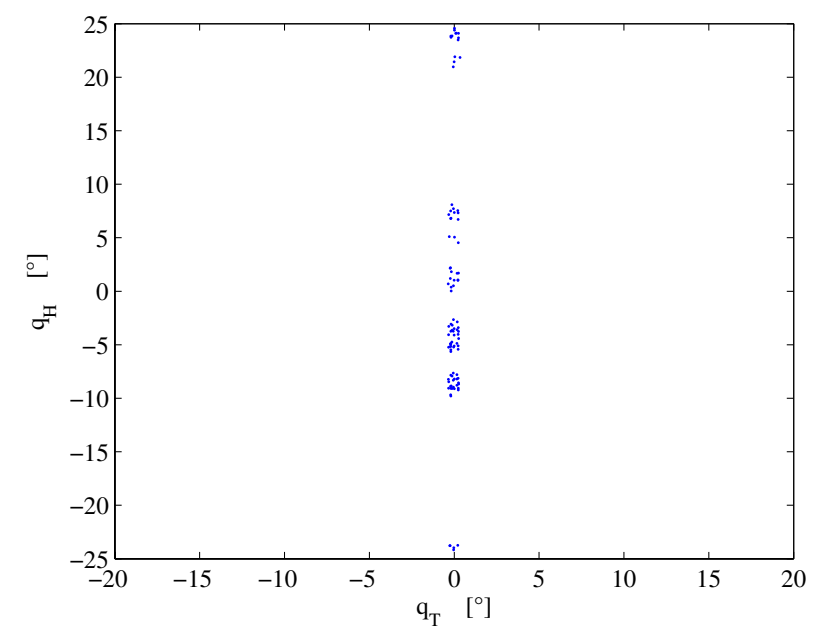

Fig. 7. Plane $\left(q_{T}, q_{H}\right)$. Each dot represents a single eye orientation. Small (random) perturbations in displacement of the PPs produce a thickening of the Listing plane

\section{B. Experimental Tests}

The goal of the experimental tests has been that of evaluating the performance of the $M A C-E Y E$ robot, in comparison with the the model proposed in the previous sections, and more important in comparison with the actual behavior of the eye in humans as documented in the literature, [5].

Since the robot eye does not feature built-in rotation sensors, an external custom-built measurement set-up has been used to measure the eye rotations. A digital camera placed in front of the eye acquires images and an automatic feature extractor tracks a set of markers placed on the eye-ball, see figure 8 .

At the beginning of each experiment the eye is placed in its nominal primary position and a calibration of the external camera is performed, [28]. After the $i-t h$ saccadic motion

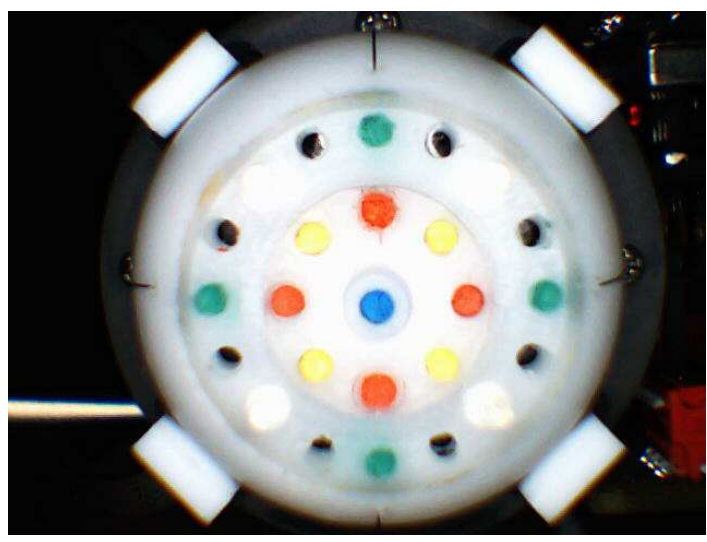

Fig. 8. Image of the eye taken from the external camera used for rotation measurements. The colored marks, placed on three different planes, are used for the automatic tracking of the eye rotations.

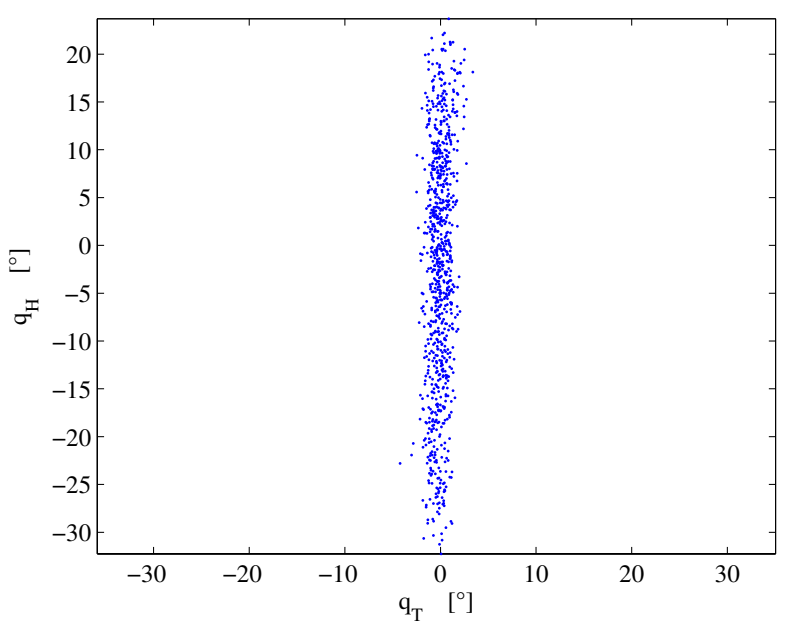

Fig. 9. Plane $\left(q_{T}, q_{H}\right)$. Each dot represents a single eye orientation. The actual eye orientations are distributed around the Listing plane. The thickness of the distribution is about $5[\mathrm{deg}]$.

an image of the eye is taken and an automatic feature tracker estimates the rotation vector $\mathbf{v}_{\mathbf{i}}$ and angle $\theta_{i}$ of the eye with respect to the nominal primary position. The Listing plane is then estimated by computing its normal unit vector $\mathbf{n}_{\mathbf{L}}$ defined as:

$$
\mathbf{n}_{\mathbf{L}}=\arg \min _{|\mathbf{n}|=1} \frac{1}{N} \sum_{i=1}^{N}\left|\mathbf{n} \cdot \mathbf{v}_{\mathbf{i}}\right|^{2}
$$

where $\mathrm{N}$ is the actual number of measurements. It is straightforward to show that $\mathbf{n}_{\mathbf{L}}$ is the unit eigenvector corresponding to the minimum eigenvalue of the semi-positive definite matrix:

$$
V_{N}=\frac{1}{N} \sum_{i=1}^{N} \mathbf{v}_{\mathbf{i}} \cdot \mathbf{v}_{\mathbf{i}}^{T}
$$

During each saccade the eye is driven by its four internal motors. Each motor is commanded to pull (or release) the corresponding tendon by an amount, computed on the basis of the nominal model of the robot eye, and specified by the desired eye rotation. Motors' rotations and tendons' tensions are controlled by the robot embedded controller.

Figure 9 shows the typical behavior of the eye described in the $\left(q_{T}, q_{H}\right)$ plane. The test corresponds to one thousand saccadic motions distributed within the whole oculomotor range. It is evident that the eye orientations approximate well the Listing's Law. As a matter of fact the actual PPs implemented in the robotic device are displaced away from the principal directions about $2[\mathrm{~mm}]$.

It is very interesting to compare the experimental data obtained from the robot with those measured on human subjects. In particular Tweed and Vilis, [5], have shown that in human subjects Listing plane has a thickness of about $5[\mathrm{deg}]$, which matches with the experimental data reported here. A typical set of measurements obtained from a human subject is shown in figure 10 (reprinted from [29]). 


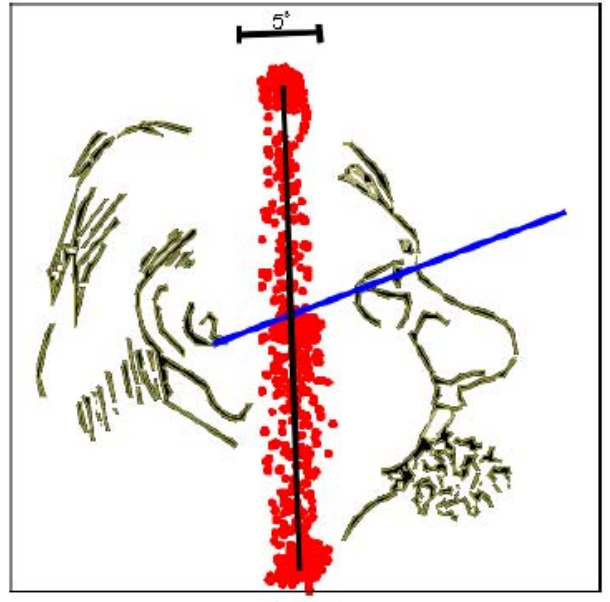

Fig. 10. Typical distribution of eye orientations in the $\left(q_{T}, q_{V}\right)$ plane, from [29]. The thickness of Listing plane is about 5 [deg].

\section{CONCLUSIONS}

In this paper we have investigated the possibility of emulating the actual saccadic motions implementing the Listing's Law on a mechanical basis. To this aim, a model of the eye plant has been proposed. The model is characterized by the relative position of the IPs on the eye-ball and of the PPs required to properly route the EOMs. Simple geometric conditions on these quantities allow to constrain the space of the moments generated by the action of the EOMs to a single plane coincident with plane $\mathcal{P}_{\omega}$. This property allows to show that any (reachable) Listing compatible eye orientation can be reached from the primary position with a trajectory composed by Listing compatible eye orientations.

Numerical simulations have validated the model. Furthermore, experimental tests have shown that the performances of a tendon driven robot eye, designed accordingly with the model specifications previously discussed, are actually quantitatively corresponding to the experimental data obtained measuring saccadic movements in human subjects.

\section{REFERENCES}

[1] G. Cannata, E. Grosso, "On Perceptual Advantages of Active Robot Vision," Journal of Robotic Systems, vol 16, n.3, 1999, pp. 163-183.

[2] W. Becker, "Eye Movements," in Carpenter, R.H.S. ed., Macmillan 1991, pp. 95-137.

[3] D. Tweed, T. Vilis, "Implications of Rotational Kinematics for the Oculomotor System in Three dimensions," The Journal of Neurophysiology, vol. 58, no.4, pp. 832-849, Oct. 1987.

[4] D. Tweed, T. Vilis, "Rotation Axes of Saccades," Ann. N. Y. Acad. Sci., vol. 545 , pp. $128-139,1988$.

[5] D. Tweed, T. Vilis, "Geometric relations of eye position and velocity vectors during saccades," Vision. Res., vol. 30, n. 1, pp. 111-127, 1990.

[6] J. M. Furman adn R. H. Schor, "Orientation of Listing's plane during static tilt in young and older human subjects," Vision Res., vol. 43, pp. 67-76, 2003.

[7] D. Straumann, D. S. Zee., D. Solomon and P. D. Kramer, "Validity of Listing's law during fixations, saccades, smooth pursuit eye movements, and blinks," Exp. Brain Res., vol. 112, pp. 135-146, 1996.

[8] G. Cannata, M. D'Andrea, F. Monti, M. Maggiali, "Implementation of Listing's Law for a Robot Eye", Proc. IFAC Symp. on Robot Control, Sy.Ro.Co 2006, Bologna (Italy), Sept. 6-8, 2006.
[9] G. Cannata, M. Maggiali, "Implementation of Listing's Law for a Tendon Driven Robot Eye", Proc. IEEE Conf. on Intelligent Robots and Systems, IROS 2006, Beijing (China), Oct. 9-15, 2006.

[10] T. Haslwanter, "Mathematics of Three-dimensional Eye Rotations," Vision Res., vol. 35, pp. 1727-1739, 1995.

[11] L. Koornneef, "The first results of a new anatomical method of approach to the human orbit following a clinical enquiry," Acta Morphol Neerl Scand, vol. 12, n. 4, pp. 259-282, 1974.

[12] J. M. Miller, "Functional anatomy of normal human rectus muscles," Vision Res., vol. 29, pp. 223-240, 1989.

[13] J. L. Demer, J. M. Miller,V. Poukens,H. V. Vinters and B.J. Glasgow, "Evidence for fibromuscular pulleys of the recti extraocular muscles," Investigative Ophthalmology and Visual Science, vol. 36, pp. 1125-1136, 1995.

[14] R. A. Clark, J.M. Miller, J. L. Demer," Three-dimensional Location of Human Rectus Pulleys by Path Inflection in Secondary Gaze Positions," Investigative Ophthalmology and Visual Science, vol. 41, pp. 3787-3797, 2000.

[15] J. L. Demer, S. Y. Ho, V. Pokens, "Evidence for Active Control of Rectus Extraocular Muscle Pulleys," Invest. Ophtalmol. Visual Sci., vol. 41, pp. 1280-1290, 2000.

[16] A. R. Koene, C.J. Erkelens, "Properties of 3D rotations and their relation to eye movement control," Biol. Cybern., vol. 90, pp. 410-417, Jul. 2004.

[17] J. Porrill, P. A. Warren, P. Dean, "A simple control law generates Listing's positions in a detailed model of the extraocular muscle system," Vision Res., vol. 40, pp. 3743-3758, 2000.

[18] C. Quaia, L. M. Optican, "Commutative Saccadic Generator Is Sufficient to Control a 3D Ocular Plant With Pulleys," The Journal of Neurophysiology, vol. 79, pp. 3197-3215, 1998.

[19] T. Raphan, "Modeling Control of Eye Orientation in Three Dimensions. I. Role of Muscle Pulleys in Determining Saccadic Trajectory," The Journal of Neurophysiology, vol. 79, pp. 2653-2667, 1998.

[20] K. Hepp, "Oculomotor control: Listing's law and all that," Current Opinion in Neurobiology, vol. 4, pp. 862-868, 1994.

[21] A. M. F. Wong, D. Tweed and J. A. Sharpe, "Adaptive Neural Mechanism for Listing's Law Revealed in Patients with Sixth Nerve Palsy," Investigative Ophtalmology and Visual Science, vol. 43, n. 1, pp. 112-118, Jan. 2002

[22] D. Biamino, G. Cannata, M. Maggiali, A. Piazza, "MAC-EYE: a Tendon Driven Fully Embedded Robot Eye", Proc. 2005 IEEE-RAS Int. Conf. on Humanoid Robots, Tsukuba, Dec. 5-7, 2005.

[23] G. K. Hung, "Models of Oculomotor Control," World Scientific Pub. Co. Inc., 2001

[24] T. Haslwanter, "Mechanics of Eye Movements: Implications of the "Orbital Revolution," Ann. N. Y. Acad. Sci., vol. 956, pp. 33-41, 2002.

[25] P. Lockwood-Cooke, C. F. Martin and L. Schovanec, "A Dynamic 3d Model of Ocular Motion," Proceedings of the 38th Conference of Decision and Control, Phoenix, Dec.1999.

[26] A. D. Polpitiya and B. K. Ghosh, "Modelling and control of eyemovement with muscolotendon dynamics," Proceedings of the American Control Conference, pp. 2313-2318, Anchorage, May, 2002.

[27] A. D. Polpitiya and B. K. Ghosh, "Modeling the Dynamics of Oculomotor System in Three Dimensions," Proceedings of the Conference on Decision and Control, pp. 6418-6422, Maui,Dec. 2003.

[28] B. Horn, "Robot Vision," Mit Press, 1986.

[29] D. Tweed, T. Vilis, "How your eyes rotate in three dimensions", Tutorial [online]. 1996 [cited 15 June 2006]. Available from: http://www.physpharm.fmd.uwo.ca/undergrad/listingweb/. 